\title{
Intrinsic GeV-TeV gamma-ray emission from EHSP blazars
}

\author{
K K Singh* \\ Department of Physics, University of the Free State, Bloemfontein 9300, South Africa \\ Astrophysical Sciences Division, Bhabha Atomic Research Centre, Mumbai 400085, India \\ E-mail: kksastro@barc.gov.in \\ P. J. Meintjes \\ Department of Physics, University of the Free State, Bloemfontein 9300, South Africa
}

\section{N. Bhatt}

Astrophysical Sciences Division, Bhabha Atomic Research Centre, Mumbai 400085, India

\section{B. van Soelen}

Department of Physics, University of the Free State, Bloemfontein 9300, South Africa

\begin{abstract}
Extremely High Synchrotron Peak (EHSP) blazars are observed to form a small population of sources with high energy hump peaking at $\mathrm{TeV}$ energies in their broad-band spectral energy distributions. The observed gamma-ray emission from these sources at GeV-TeV energies is described by unusual hard spectral indices. The observed spectral and temporal characteristics of these sources challenge the standard leptonic models for the broad-band emissions from blazars. Therefore, such sources provide astrophysical sites to investigate directly the particle acceleration, cooling of relativistic particles and indirectly probe the cosmological quantities like extragalactic background light (EBL) and intergalactic magnetic field (IMF) in the Universe. In this study, we investigate the spectral properties of the gamma-ray emission from EHSP blazars using observations from the Fermi-LAT catalogues (3FGL and 3FHL) along with the TeV observations using ground based telescopes. The observed $\mathrm{TeV}$ gamma-ray spectra are corrected for the EBL absorption using the most recent and updated EBL models to determine the intrinsic spectrum at the source. The intrinsic $\mathrm{TeV}$ spectra are combined with the $\mathrm{MeV}-\mathrm{GeV}$ observations from the Fermi-LAT to study the gamma- ray emission from EHSP blazars in the broad energy band. The intrinsic gamma-ray spectra are then used to estimate the position of high energy peak in the spectral energy distribution. We also present the qualitative description for the observed spectral properties of EHSP blazars using different physical scenarios and discuss their importance for the upcoming CTA observatory.
\end{abstract}

36th International Cosmic Ray Conference -ICRC2019-

July 24th - August 1st, 2019

Madison, WI, U.S.A.

\footnotetext{
${ }^{*}$ Corresponding Author
} 


\section{Introduction}

Blazars are a dominant class of radio-loud active galactic nuclei (AGN) which emit nonthermal radiation over the entire electromagnetic spectrum from radio to very high energy (VHE; $\mathrm{E}>50 \mathrm{GeV}$ ) gamma rays. The observed broadband radiation from blazars is interpreted as the multi-wavelength emission from the relativistic plsama jet which points in the direction of the observer at small viewing angle [1]. The spectral energy distribution (SED) of blazars is described by two characteristic humps peaking at low (IR/optical/X-ray) and high ( $\gamma$-ray) energies respectively. The physics of the low energy hump in the blazar SED is well established and is attributed to the synchrotron emission from the relativistic leptons (mostly electrons and positrons) in the jet magnetic field. On the basis of the position of rest frame synchrotron peak frequency $\left(v_{\text {peak }}^{\text {sync }}\right)$ in the SED, blazars are classified as low synchrotron peaked (LSP: $v_{\text {peak }}^{\text {sync }}<10^{14} \mathrm{~Hz}$ ), intermediate synchrotron peaked (ISP: $10^{14} \mathrm{~Hz}<v_{\text {peak }}^{\text {sync }}<10^{15} \mathrm{~Hz}$ ), high synchrotron peaked (HSP: $10^{15}$ $\mathrm{Hz}<v_{\text {peak }}^{\text {syn }}<10^{17} \mathrm{~Hz}$ ) and extremely high synchrotron peaked (EHSP: $v_{\text {peak }}^{\text {sync }}>10^{17} \mathrm{~Hz}$ ) blazars $[2,3]$. The mechanism of $\gamma$-ray emission and origin of high energy (HE; E $>0.1 \mathrm{GeV}$ ) hump in the SED are still under debate in blazar research. In general, $\gamma$-ray photons at $\mathrm{GeV}-\mathrm{TeV}$ energies are supposed to be produced by the inverse Compton (IC) scattering of low energy photons by the relativsitic leptons in the blazar jet under the frame-work of leptonic models [4, 5]. Alternatively, in a hadronic scenario, proton synchrotron and photo-hadronic interactions are also invoked to explain the observed $\gamma$-ray emission from blazars [6, 7]. Leptonic and Lepto-hadronic (Hybrid) models have been successfully used to describe the VHE $\gamma$-ray emission from most of the blazars observed so far. Variability in the spectrum and light curves in almost all energy bands at different timescales ranging from few minutes to years is observed to be a common feature of blazars [8,9]. However, EHSP sources challenge the $\gamma$-ray emission models proposed for blazars and lack rapid flux variability in $\gamma$-ray energy band [10]. In this study, we use a sample of four well known EHSP blazars to understand the $\gamma$-ray emission processes from these peculiar sources.

\section{VHE $\gamma$-ray Emission from EHSP Blazars}

The intrinsic VHE $\gamma$-ray emission observed from EHSP blazars is described by a power law with hard photon spectral index $\Gamma_{\text {int }} \leq 2$. Due to their hard spectra, the peak of $\gamma$-ray component in the broadband SED is located at energies above $1 \mathrm{TeV}$. This challenges the standard leptonic emission models based on IC scattering of synchrotron photons or external photons for $\gamma$-ray emission from the blazars. The IC scattering in the Thomson regime decreases with increasing electron energy due to the lower energy density of target photons in the emission region of the jet. In the Klien-Nishina (KN) regime, the spectrum of VHE $\gamma$-rays is steeper or soft due to a decrease in the efficiency of the IC scattering [10]. The hard $\gamma$-ray spectra also indicate injection of relativistic particles having very hard spectrum with index less than 1.5 in the emission region of the jet. This poses a strong challenge to the accelearion of particles through diffusive shock acceleration in the jet. Therefore, following alternative scenarios including hadronic processes have been invoked to explain the gamma-ray emission from the EHSP blazars:

- IC scattering of synchrotron photons by the relativsitic electrons in the emission region under the frame-work of a simple one zone leptonic model can produce VHE $\gamma$-rays with extremely 
hard spectra assuming large Doppler factor values as well as very high values of minimum Lorentz factor of the electrons with low radiative efficiency, and physical conditions far way from the equipartion [11]. Large Doppler factors indicate emission region moving relativistically at small viewing angle which is not consistent with radio observations of the knots in the jet.

- IC scattering of low energy seed photons by a narrow energetic particle distribution (relativistic Maxwellian produced by a stochastic Fermi-second order acceleration) in the Thomson regime can emit hard $\mathrm{TeV}$ spectra [12]. Also within the frame-work of single zone leptonic models, a power law distribution with a low energy cutoff of electrons in VHE band can in principle produce hard $\gamma$-ray spectra.

- IC scattering of cosmic microwave background (CMB) photons by the ultra-relativistic electrons produced by the shock acceleration in an extended jet (kpc-scale) can result in the slowly variable VHE $\gamma$-ray emission with very hard spectrum in the Thomson limit [13]. This scenario suggests separate components for X-ray and $\gamma$-ray emissions from the blazar.

- Internal $\gamma-\gamma$ absorption of VHE $\gamma$-rays due to their interaction with dense narrow-band low energy radiation fields close to the emission region in the jet can lead to the formation of $\mathrm{TeV}$ spectra with arbitrary hardness in a natural way [14]. This does not require any modification in the particle spectra or particle acceleration models.

- Lepto-hadronic processes including IC scattering of synchrotron photons produced by leptons, proton synchrotron and synchrotron emission from the cascade initiated by the proton- $\gamma$ interaction can collectively produce hard $\gamma$-ray spectra from blazars [15].

- In a completely different scenario for EHSP blazars, $\gamma$-rays are not produced in the jet, instead in the intergalactic space. The ultra-high energy cosmic rays (UHECRs) escaping from the jet (mostly protons with energy above $10^{18} \mathrm{eV}$ ) and beamed towards the observer due to weak cosmic magnetic field, interact with the CMB or UV/IR/optical photons through photo-meson and pair production in the intergalactic medium and initiate development of electromagnetic cascade. The $\gamma$-rays with hard spectra are produced by the secondary particles in the cascade [16]. Recent detection of high energy astrophysical neutrinos from a few blazars support this hypothesis for VHE $\gamma$-ray production.

Therefore, the EHSP blazars with hard VHE $\gamma$-ray spectra provide a unique opportunity to explore many physical processes in high energy astrophysics. These sources are ideal candidates for probing the jet physics, particle acceleration of UHECRs, intergalactic magnetic field (IGMF) and density of low energy background photons from $\mathrm{CMB}$ to optical wavelengths in the intergalactic space.

\section{EHSP Candidates}

The EHSP blazars are also known as hard spectra blazars. Due to their hard $\gamma$-ray spectra, the EHSP blazars are faint in the energy range $(0.1-500 \mathrm{GeV})$ covered by the Large Area Telescope (LAT) onboard the Fermi satellite. However, a small group of this new and emerging class 


\begin{tabular}{lclccc}
\hline Name & Redshift $(z)$ & $\Gamma_{3 F G L}$ & $\Gamma_{3 F H L}$ & $\Gamma_{T e V}$ & VHE Observation \\
\hline RGB J0710+591 & 0.125 & $1.661 \pm 0.094$ & $1.022 \pm 0.370$ & $2.64 \pm 0.27$ & VERITAS [19] \\
1ES 0229+200 & 0.140 & $2.025 \pm 0.150$ & $1.541 \pm 0.401$ & $2.61 \pm 0.14$ & VERITAS [20] \\
1ES 0347-121 & 0.188 & $1.734 \pm 0.156$ & $1.830 \pm 0.290$ & $3.09 \pm 0.20$ & H.E.S.S. [21] \\
1ES 0414+009 & 0.287 & $1.745 \pm 0.114$ & $1.851 \pm 0.337$ & $3.43 \pm 0.58$ & VERITAS [22] \\
\hline
\end{tabular}

Table 1: Summary of the results from the $\gamma$-ray observations of the ESHP blazar candidates.

of blazars have been detected in $\mathrm{GeV}$ energy range by the Fermi-LAT and at $\mathrm{TeV}$ energies by the current generation ground based Imaging Air Chererenov Telescopes (IACTs) like VERITAS, MAGIC and H.E.S.S. . The results from the long term Fermi-LAT observations are reported in its different $\gamma$-ray catalogs. In this study, we have selected four well known EHSP candidate sources for which quasi-simultaneous observations from the Fermi-LAT and IACTs are available. In the Third Fermi-LAT source catalog (3FGL), time averaged spectral measurements in the energy range 0.1-100 GeV have been reported using four years of science data collected during the period August 2008- July 2012 [17]. The Third Catalog of Hard Fermi-LAT sources (3FHL) provides results above $10 \mathrm{GeV}$ from the seven years of observations between August 2008 to August 2015 [18]. This indicates that the Fermi-LAT observations of the HE $\gamma$-ray sky provide an excellent overlap with the ground based VHE observations since its scientific operation began in 2008. The differential spectra of $\gamma$-ray emission from the EHSP blazars measured using Fermi-LAT or IACTs are described by a power law of the form:

$$
\frac{d N}{d E}=N_{0}\left(\frac{E}{E_{0}}\right)^{-\Gamma}
$$

where $N_{0}$ is the normalization flux at the scale (pivot) energy $E_{0}$ and $\Gamma$ is the photon spectral index. The values of spectral indices reported in in the 3FGL $\left(\Gamma_{3 F G L}\right)$ and $3 \mathrm{FHL}\left(\Gamma_{3 F H L}\right)$ along with the VHE observations $\left(\Gamma_{T e V}\right)$ for four ESHP candidates selected in the present study are reported in Table 1. It is evident from Table 1 that the observed $\mathrm{TeV}$ spectral indices are significantly softer than the Fermi-LAT spectral indices in GeV energy band.

\section{Results and Discussion}

\subsection{Intrinsic VHE Spectrum}

The VHE $\gamma$-ray observations above $100 \mathrm{GeV}$ by ground based telescopes suffer an opacity effect due to the presence of Extragalactic Background Light (EBL) in the intergalactic space. The EBL consists of IR-optical-UV photons emitted by the stars and galaxies throughout the evolution of the Universe. VHE $\gamma$-rays emitted from the cosmic sources are absorbed by the low energy $\left(10^{-3}\right.$ $\mathrm{eV}-10 \mathrm{eV}) \mathrm{EBL}$ photons via $\gamma-\gamma$ pair production. This process can partially remove the VHE photons from the beam propogating towards the observer and causes opacity to $\mathrm{TeV}$ observations of the sources in the extragalactic Universe. The opacity of the Universe is characterized by the optical-depth $(\tau)$ which strongly depends on the redshift of the source $(z)$, energy of VHE $\gamma$-rays $(E)$ and density of the EBL photons. The method of computing $\tau(E, z)$ for a given model of EBL 


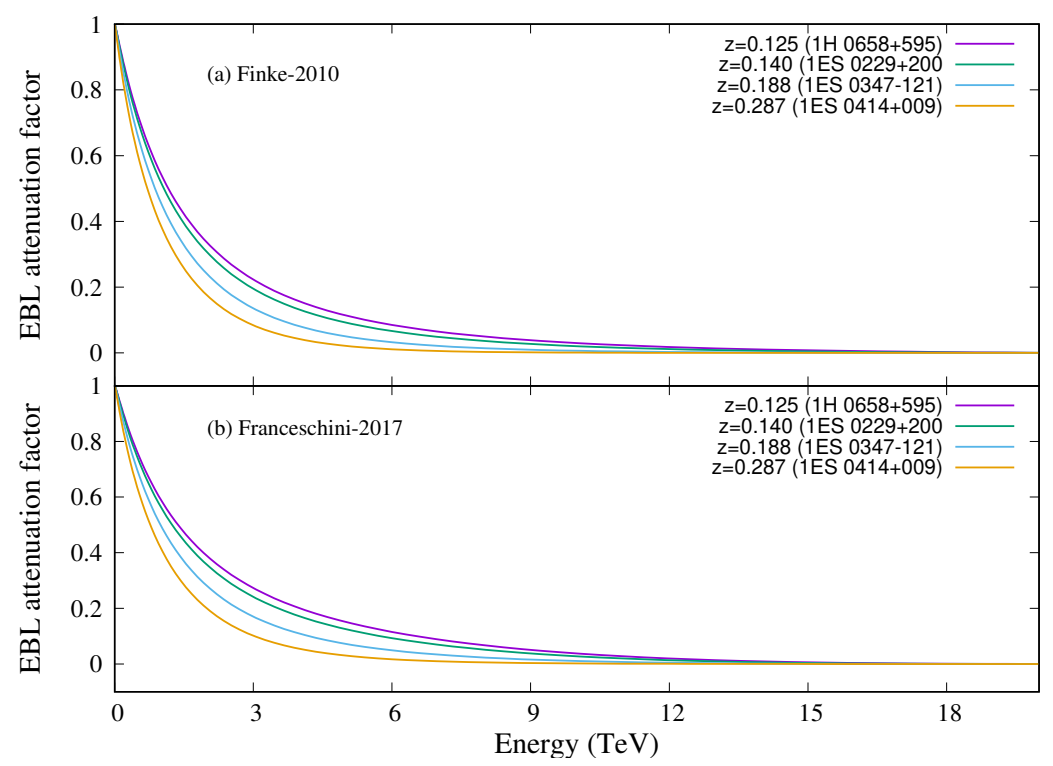

Figure 1: Attenuation of $\gamma$-ray photons due to EBL absorption for sources at various redshifts using two different EBL models proposed by (a) Finke et al. (2010) and (b) Franceschini et al. (2017).

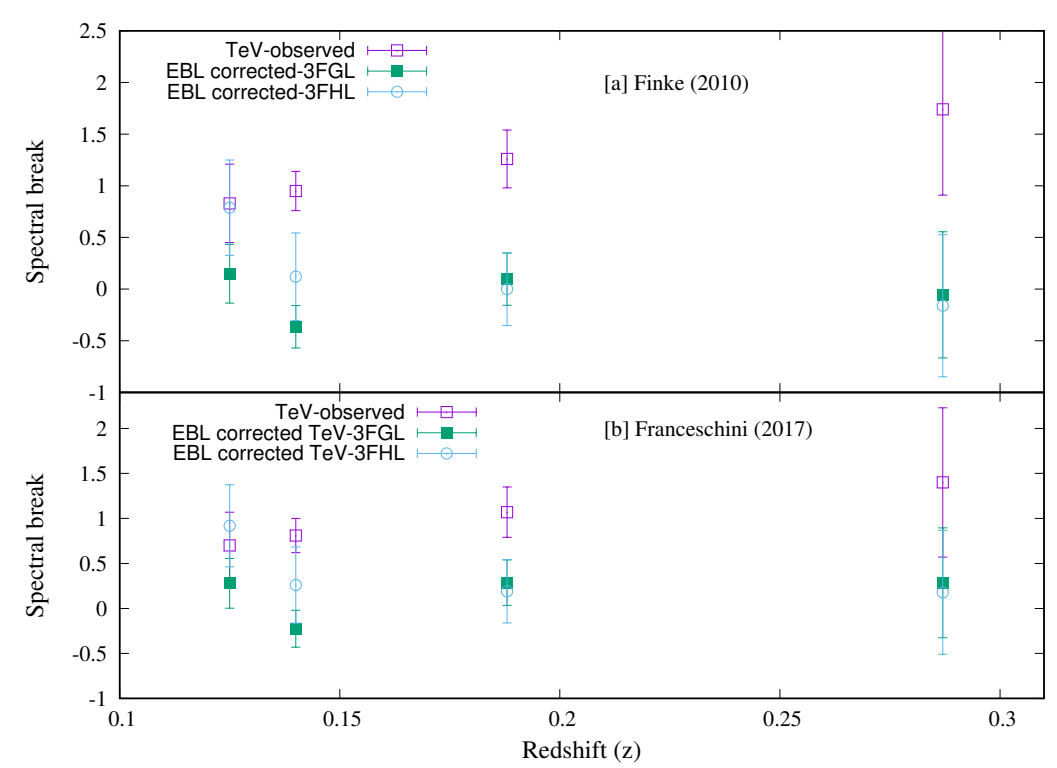

Figure 2: Spectral Break between $\Gamma_{i n t}$ and $\Gamma_{T e V}, \Gamma_{3 F G L} \& \Gamma_{3 F H L}$ for EHSP candidate blazars using (a) Finke et al. (2010) and (b) Franceschini et al. (2017) models.

photon density is described in [23]. The VHE $\gamma$-ray spectrum of a source observed by the ground based instruments is modified with respect to the intrinsic spectrum and both are related as:

$$
\left(\frac{d N}{d E}\right)_{o b s}=\left(\frac{d N}{d E}\right)_{i n t} e^{-\tau(E, z)}
$$

where the term $\mathrm{e}^{-\tau(E, z)}$ is commonly referred to as EBL attenuation factor. The EBL attenuation factor as a function of energy in the range $0.1 \mathrm{GeV}$ to $20 \mathrm{TeV}$ for four ESHP blazars at different $z$ is shown in Figure 1 (a) \& (b) corresponding to the two widely used EBL models proposed by Finke 

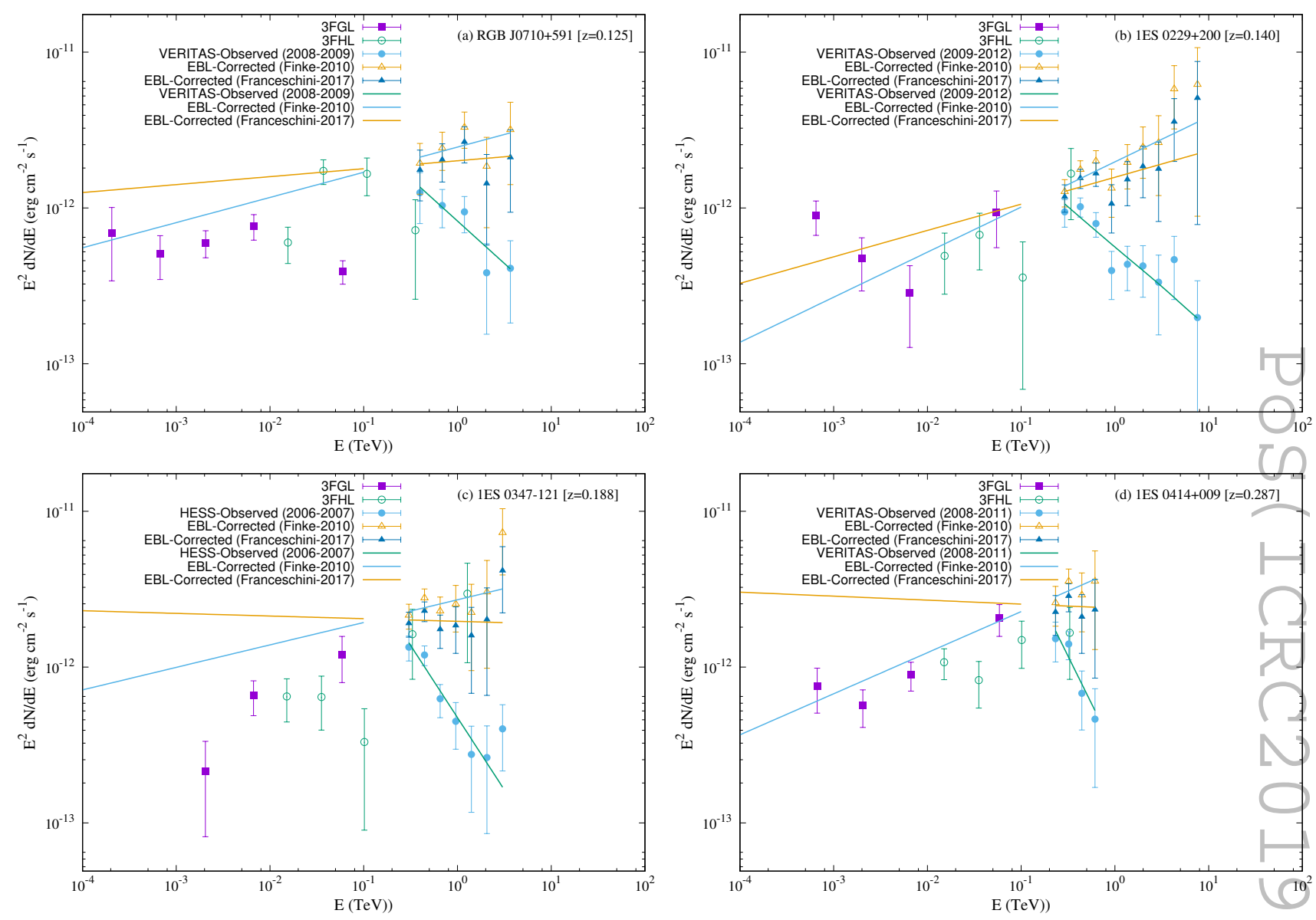

Figure 3: GeV-TeV spectral energy distribution of EHSP blazars.

et al. (2010) [24] and Franceschini et al. (2017) [25] respectively. We observe that the attenuation due to EBL for both the models is negligible up to $100 \mathrm{GeV}$ for all sources considered in the present study. However, $\mathrm{TeV} \gamma$-ray photons above $100 \mathrm{GeV}$ suffer large EBL absorption. This can lead to the significant softening of the intrinsic VHE spectra emitted from the sources at different redshifts. We have derived the intrinsic $\mathrm{TeV}$ spectra for all the sources from the observed flux points after correcting for EBL absorption (Equation 4.1) using two different models. The intrinsic TeV spectra are also described by a power law with spectral indices $\Gamma_{i n t}$ which are siginificantly harder than the observed spectral indices $\Gamma_{T e V}$ reported in Table 1.

\subsection{GeV-TeV Spectral Break and Spectral Energy Distribution}

The absorption of VHE $\gamma$-rays due to interaction with EBL suggests that observed TeV spectrum of a source is different from the intrinsic $\mathrm{TeV}$ spectrum and the $\mathrm{GeV}$ spectrum measured from the Fermi-LAT. The difference between any two power law spectral indices is referred to as spectral break. We have estimated the spectral break caused by the absorption of $\mathrm{TeV}$ photons for the EHSP candidate blazars (Table 1) using two different EBL models and the results are shown in Figure 2 (a) \& (b) as a function of redshift. It is found that the spectral break between observed and intrinsic TeV spectra $\left(\Gamma_{\mathrm{TeV}}-\Gamma_{\text {int }}\right)$ increases with redshift whereas the break between the Fermi-LAT 
spectral indices $\left(\Gamma_{3 F G L} \& \Gamma_{3 F H L}\right)$ and $\Gamma_{\text {int }}$ is nearly zero for all redshifts.

The SED of four EHSP blazars in the energy range $0.1 \mathrm{GeV}$ to few $\mathrm{TeV}$ are shown in Figure 3(a-d). The intrinsic TeV spectra of all the sources estimated using the EBL model proposed by Finke et al. 2010 [24], are described by a power law with an average photon spectral index $\Gamma_{\text {int }} \sim 1$.7. This is broadly consitent with average of corresponding $\Gamma_{3 F G L}$ and $\Gamma_{3 F H L}$ values. The extrapolation of the intrinsic $\mathrm{TeV}$ spectrum following a power law in the $\mathrm{GeV}$ energy band suggests that the HE flux points measured by the Fermi-LAT are generally below the flux level expected from the VHE observations. For 1ES 0347-121, the HE emission in the range $100 \mathrm{MeV}$ to $1 \mathrm{GeV}$ is below the detection sensitivity of the Fermi-LAT and deviation of the flux points above $1 \mathrm{GeV}$ from the $\mathrm{TeV}$ extraploation is also relatively large. The differential spectra of $\gamma$-ray photons with hard spectral index $\sim 1.7$ suggest that the HE component of SED peaks at TeV energies. As discussed in Section 2, such hard $\mathrm{TeV}$ spectra can be mostly produced by the various electron distributions like hard power law, low-energy cutoff or Maxwellian with tail at higher energies. These spectral features in the electron distribution should also be observed in the synchrotron spectrum at low energies up to X-rays as synchrotron radiation is assumed to be produced by the same population of electrons in the frame-work of simple leptonic models. However, the synchrotron components in the broad-band SED of EHSP candidate blazars do not show such spectral features. This indicates that hadronic processes are the possible explanation for $\mathrm{GeV}-\mathrm{TeV}$ emission from these sources.

\section{Conclusions}

The intrinsic $\mathrm{TeV}$ spectral indices of EHSP candidates are in good agreement with the Fermi-LAT spectra. However, the flux points measured from the Fermi-LAT are below the extrapolated $\mathrm{TeV}$ flux levels for all the sources considered in the present study. The combined $\mathrm{GeV}-\mathrm{TeV} \gamma$-ray spectra of all the EHSP candidates are described by a single power law with photon spectral index $\Gamma \sim 1.7$ which is consistent with the proton synchrotron radiation due to injection spectral index of $\sim 2.4$ in the emission region. However, simultaneous long term monitoring of these sources in a wide $\mathrm{GeV}-\mathrm{TeV}$ energy range by the upcoming Cherenkov Telescope Array (CTA)-observatory will be very important to fully explore the $\gamma$-ray emission processes from this new class of blazars. This will consequently help in constraining the EBL photon density permeated in the intergalactic space.

\section{References}

[1] P. Padovani et al., Active galactic nuclei: what's in a name?, A\&ARv 25 (2017) 2 [arXiv:1707.07134]

[2] L. Costamante et al., Extreme synchrotron BL Lac objects. Stretching the blazar sequence, A\&A $\mathbf{3 7 1}$ (2001) 512 [astro-ph/0103343]

[3] A. A. Abdo et al., The Spectral Energy Distribution of Fermi Bright Blazars, ApJ 716 (2010) 30 [arXiv:0912.2040]

[4] M. Böttcher, Modeling the emission processes in blazars, Ap\&SS 309 (2007) 95 [astro-ph/0608713]

[5] I. Agudo et al., On the Location of the $\gamma$-Ray Outburst Emission in the BL Lacertae Object AO 0235+164 Through Observations Across the Electromagnetic Spectrum, ApJL 735 (2011) 10 [arXiv:1105.0549] 
[6] F. A. Aharonian, Proton-synchrotron radiation of large-scale jets in active galactic nuclei, MNRAS 332 (2002) 215 [astro-ph/ 0106037 ]

[7] M. Böttcher et al., Leptonic and Hadronic Modeling of Fermi-detected Blazars, ApJ 768 (2013) 54 [arXiv:1304.0605]

[8] J. Albert et al., Variable Very High Energy $\gamma$-Ray Emission from Markarian 501, ApJ 669 (2007) 862 [astro-ph/0702008]

[9] K. K. Singh et al., Study of short term enhanced TeV $\gamma$-ray emission from Mrk 421 observed with TACTIC on December 28, 2014 APh 103 (2018) 122 [arXiv: 808.02670 ]

[10] K. K. Singh et al., Extremely High energy peaked BL Lac nature of the TeV blazar Mrk 501, NewA 73 (2019) 101278 [arXiv:1906.04486]

[11] L. Costamante et al., The NuSTAR view on hard-TeV BL Lacs, MNRAS 477 (2018) 4257 [arXiv:1711.06282]

[12] E. Lefa et al., Formation of Very Hard Gamma-Ray Spectra of Blazars in Leptonic Models, ApJ 740 (2011) 64 [arXiv:1106.4201]

[13] M. Böttcher et al., The Hard VHE $\gamma$-Ray Emission in High-Redshift TeV Blazars: Comptonization of Cosmic Microwave Background Radiation in an Extended Jet ?, ApJL 679 (2008) 9 [arXiv:0804.3515]

[14] F. A. Aharonian et al., Formation of hard very high energy gamma-ray spectra of blazars due to internal photon-photon absorption, MNRAS 387 (2008) 1206 [arXiv: 0801 . 3198]

[15] M. Cerruti et al., A hadronic origin for ultra-high-frequency-peaked BL Lac objects, MNRAS 448 (2015) 910 [arXiv: 1411.5968 ]

[16] W. Essey et al., Role of Line-of-sight Cosmic-ray Interactions in Forming the Spectra of Distant Blazars in TeV Gamma Rays and High-energy Neutrinos, ApJ 731 (2011) 51 [arXiv: 1011.6340 ]

[17] F. Acero et al., Fermi Large Area Telescope Third Source Catalog, ApJS 218 (2015) 23 [arXiv:1501.02003]

[18] M. Ajello et al., 3FHL: The Third Catalog of Hard Fermi-LAT Sources, ApJS 232 (2017) 18 [arXiv:1702.00664]

[19] V. A. Acciari et al., The Discovery of $\gamma$-Ray Emission from the Blazar RGB J0710+591, ApJL 715 (2010) 49 [arXiv: 1005.0041$]$

[20] E. Aliu et al., A Three-year Multi-wavelength Study of the Very-high-energy $\gamma$-Ray Blazar IES 0229+200, ApJ 782 (2014) 13 [arXiv: 1312 . 6592]

[21] F. A. Aharonian et al., Discovery of VHE $\gamma$-rays from the distant BL Lacertae 1ES 0347-121, A\&A 473 (2007) 25 [arXiv: 0708.3021$]$

[22] E. Aliu et al., Multiwavelength Observations of the AGN 1ES 0414+009 with VERITAS, Fermi-LAT, Swift-XRT, and MDM, ApJ 755 (2012) 118 [arXiv: 1206.4080 ]

[23] K. K. Singh et al., Intrinsic VHE gamma-ray spectra of blazars as a probe for extragalactic background light, NewA 27 (2014) 34 [arXiv:1310 . 8386]

[24] J. D. Finke et al., Modeling the Extragalactic Background Light from Stars and Dust, ApJ 712 (2010) 238 [arXiv:0905.1115]

[25] A. Franceschini et al., The extragalactic background light revisited and the cosmic photon-photon opacity, A\&A $\mathbf{6 0 3}$ (2017) 34 [arXiv: 1705.10256 ] 